\title{
The isolate Caproiciproducens sp. 7D4C2 produces $n$-caproate at mildly acidic conditions from hexoses: genome and rBOX comparison with related strains and chain-elongating bacteria
}

Sofia Esquivel-Elizondo*,a, Caner Bağcl, ${ }^{b, c}$, Monika Temovska d, Byoung Seung Jeon ${ }^{d}$, Irina Bessarab ${ }^{e}$, Rohan B. H. Williams ${ }^{e}$, Daniel H. Huson ${ }^{b}$, and Largus T. Angenent ${ }^{a, d}$

* presenter, sofia.esquivel-elizondo@tuebingen.mpg.de

a AG Angenent, Max Planck Institute for Developmental Biology, Germany;

${ }^{b}$ Algorithms in Bioinformatics, Department of Computer Science, University of Tübingen, Germany; ${ }^{c}$ International Max Planck Research School "From Molecules to Organisms", Max Planck Institute for Developmental Biology and University of Tübingen, Germany; ${ }^{d}$ Environmental Biotechnology Group, Center for Applied Geosciences,

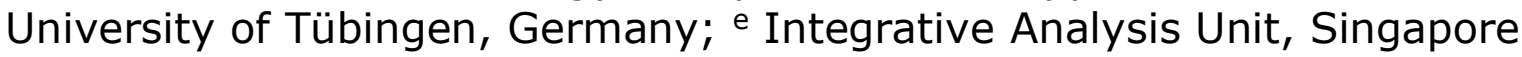
Centre for Environmental Life Sciences Engineering, National University of Singapore, Singapore.

\section{HIGHLIGHTS:}

- We isolated a chain-elongating bacterium that thrives at mildly acidic $\mathrm{pH}$ levels.

- We assembled its complete genome, which we used to assign taxonomy and to study its rBOX genes.

- The rBOX genes of the Caproiciproducens spp. are conserved and located next to each other, forming a gene cluster.

BACKGROUND: Bulk production of medium-chain carboxylates (MCCs) with 6-12 carbon atoms is of great interest to biotechnology. Open cultures (e.g., reactor microbiomes) have been utilized to generate MCCs in bioreactors. When in-line MCC extraction and prevention of product inhibition is required, the bioreactors have been operated at mildly acidic $\mathrm{pH}$ (5.0-5.5). However, model chain-elongating bacteria grow optimally at neutral $\mathrm{pH}$ values.

RESULTS \& DISCUSSION: We isolated a chain-elongating bacterium (strain 7D4C2) that thrives at mildly acidic $\mathrm{pH}$. We studied its metabolism and compared its whole genome and the reverse $\beta$-oxidation (rBOX) genes to other bacteria. Strain 7D4C2 produces lactate, acetate, $n$-butyrate, $n$ caproate, biomass, and $\mathrm{H}_{2} / \mathrm{CO}_{2}$ from hexoses. With only fructose as substrate $(\mathrm{pH} 5.5)$, the maximum $n$-caproate specificity (i.e., products per other carboxylates produced) was $60.9 \pm 1.5 \%$. However, this was 
considerably higher at $83.1 \pm 0.44 \%$ when both fructose and $n$-butyrate (electron acceptor) were combined as a substrate. A comparison of serum bottles with fructose and $n$-butyrate with an increasing $\mathrm{pH}$ value from 4.5 to 9.0 showed a decreasing $n$-caproate specificity from $\sim 92 \%$ at mildly acidic $\mathrm{pH}(\mathrm{pH} 4.5-5.0)$ to $\sim 24 \%$ at alkaline $\mathrm{pH}(\mathrm{pH} 9.0)$. Moreover, when carboxylates were extracted from the broth (undissociated $n$-caproic acid was $\sim 0.3 \mathrm{mM}$ ), the $n$-caproate selectivity (i.e., product per substrate fed) was $42.6 \pm 19.0 \%$ higher compared to serum bottles without extraction. Based on the 16S rRNA gene sequence, strain 7D4C2 is most closely related to the isolates Caproicibacter fermentans (99.5\%) and Caproiciproducens galactitolivorans (94.7\%), which are chain-elongating bacteria that are also capable of lactate production. Whole-genome analyses indicate that strain 7D4C2, C. fermentans, and C. galactitolivorans belong to the same genus of Caproiciproducens. Their rBOX genes are conserved and located next to each other, forming a gene cluster, which is different than for other chainelongating bacteria such as Megasphaera spp (Figure 1).

CONCLUSION: Caproiciproducens spp., comprising strain 7D4C2, C. fermentans, C. galactitolivorans, and several unclassified strains, are chainelongating bacteria that encode a highly conserved rBOX gene cluster. Caproiciproducens sp. 7D4C2 (DSM 110548) was studied here to understand $n$-caproate production better at mildly acidic $\mathrm{pH}$ within microbiomes and has the additional potential as a pure-culture production strain to convert sugars into $n$-caproate.

A)

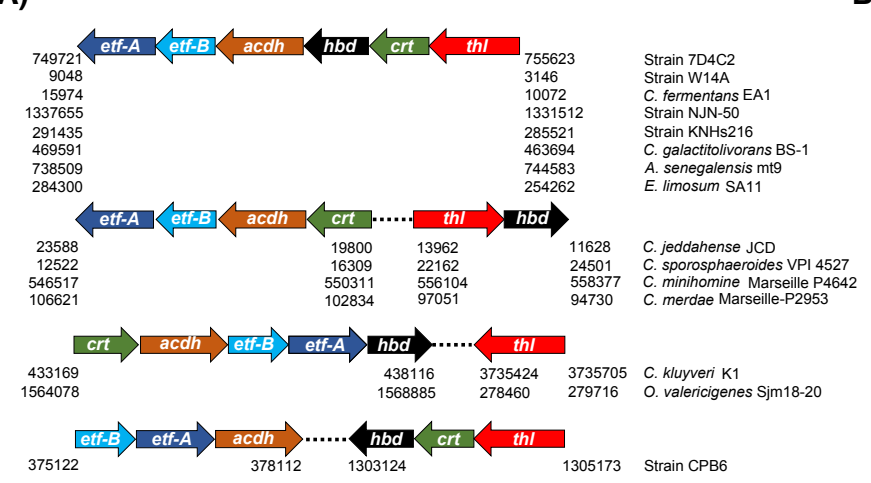

B)

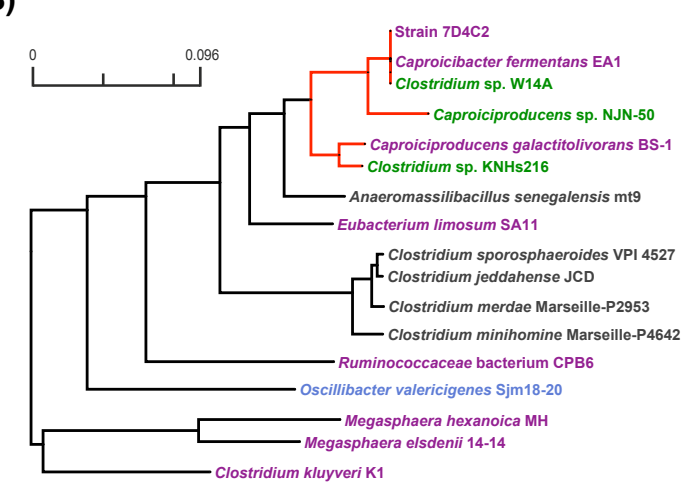

Figure 1. rBOX genes for strain 7D4C2 and bacteria with similar genes, as well as in known $n$-caproate producers: A) position of the rBOX genes that cluster together in these bacteria. The numbers below the arrows indicate the position (base pairs) of the genes for each bacterium on the right column; and B) consensus phylogenetic tree of all 6 rBOX genes that cluster together*. Red lines indicate the Caproiciproducens clade. Microbial names highlighted in purple denote $n$-caproate producers, in green are potential $n$ caproate producers, and in blue $n$-valerate producers. *As the rBOX genes in the Megasphaera species do not cluster, for this analysis, we considered the genes most similar to strain 7D4C2. 